\title{
Research on Key Technologies and Applications of Energy Internet Blockchain
}

\author{
Xingxiong $\mathrm{Zhu}{ }^{1,2, *}$ \\ ${ }^{1}$ State Grid Power Finance and E-Commerce Laboratory, 100053 Beijing, China \\ 2 State Grid Electronic Commerce Co., Ltd., 100053 Beijing, China
}

\begin{abstract}
In view of the challenges facing the current development of energy Internet, the basic theory, architecture implementation and application mode of energy blockchain technology are proposed from the communication layer, data layer and contract layer. In this paper, we analyze the value distribution mode of blockchain in energy internet, integrate business form, information system, Internet of Things and blockchain, and build secure and autonomous peer-to-peer energy Internet environment based on blockchain. The key technologies are constructed from the network layer, system layer and business layer. The problems of network layer equipment and data access authentication, secure and trusted sharing are solved. The system layer blockchain-based power payment, power settlement, power audit and other business support capabilities are improved. The system enhances the security and credibility of business, and drives the future global energy Internet development.
\end{abstract}

\section{Introduction}

The energy Internet takes the power system as the core, utilizes the Internet and other cutting-edge information technologies, and uses distributed renewable energy as the primary energy source, which is the energy utilization system [1].

Due to the technical level and application level, the application of blockchain-based energy Internet technology is still in its infancy. Therefore, research on the key technologies of blockchain for typical applications of energy Internet, study the core technologies of energy Internet and blockchain, expand the application of blockchain technology in the energy Internet, build unified payment prototype system based on blockchain, and innovate the traditional Internet landscape and model.

As an autonomous and reliable network technology, blockchain has important theoretical and practical value. A secure and autonomous peer-to-peer network platform is built to ensure the effective transmission of energy Internet values. A settlement method is provided to support final audits and improve the credibility of transactions such as power payment. The system ensures the security and credibility of Internet of Things (IoT) devices and data from source to data sharing, supports the development of energy Internet finance, and realizes the storage of data on grid transactions based on blockchain.

\section{Key technologies for energy blockchain}

\footnotetext{
* Corresponding author: zhuxx@pku.org.cn
}

\subsection{Energy Internet value chain based on blockchain technology}

A value transfer model and role relationship model are established for energy blockchain technology in energy industry. In this paper, we face the new things in the energy field of energy Internet, combine the advantages of energy Internet and blockchain technology, jointly solve the existing pain points in the energy industry, and improve the quality and efficiency of the energy Internet value chain.

\subsection{Blockchain-based energy finance business}

The blockchain-based financial service fusion method is different from the traditional data storage method which based on traditional database [2]. The blockchain-based financial service is based on distributed blocks, and the existing business logic is realized on the structure of distributed blocks.

The traditional approach is to use centralized data center and in-memory database. However, blockchainbased financial services have high anonymity and realtime performance.

\subsection{Power payment based on blockchain technology}

The hierarchical structure model of charging pile unified payment based on alliance blockchain is constructed to form a theoretical system and technical framework. 
A secure and efficient unified payment mechanism based on the charging pile alliance chain is constructed. Automated transactions and financial settlement based on smart contracts, and achieves unified payment for heterogeneous node consensus are supported. The barrier-free payment of electric vehicles nationwide is solved, and payment efficiency is optimized.

\subsection{Peer-to-peer independent power settlement technology based on blockchain}

In combination with the power settlement service, a private block data structure suitable for settlement business is designed. The key to intelligent contract layer is writing and parsing of intelligent contracts based on blockchain to ensure the uniform execution of contract codes. Intelligent, common execution bytecodes and interpreters are required between nodes in the blockchain. Computational nodes can achieve consistency when executing contracts. Power digital currency-based power transaction construction and financial settlement are implemented at the settlement business layer.

In the data layer, contract layer, business layer: verify the effectiveness of the private chain structure and the efficiency of transaction query in the data layer. At the contract level, because the smart contract is executed on the blockchain, the result of the execution is also stored in the blockchain. The flexibility of contract execution is required to control the reading of the blockchain data. At the business level, power transactions are mapped to a combination of power coins and smart contracts.

\subsection{Auditing methods and techniques based on blockchain technology}

Using the time-series, distributed and trusted features of the blockchain technology, we construct a block storage structure method conforming to the audit data standard, a data trust method based on the digital signature technology and the digital summary technology. Blockchain-based high-speed query method, time-stampbased blockchain recording, and time synchronization techniques for each block are constructed. The method of audit smart contract is constructed to improve the audit work efficiency and the correctness of the results due to the inconsistency of data and unreliable data during the audit process.

The distributed nature of blockchain technology, combined with the demand characteristics of auditing services, allows block of data to be used by different auditing services from different dimensions. Therefore, the storage standard of block data needs to be clarified. The time consistency of each block in the blockchain is ensured [3]. In this paper, we optimize of block data query speed, model the audit business and then merge with blockchain-based smart contracts.

\subsection{Data security sharing system based on blockchain technology}

A reliable, efficient, and low-cost hybrid chain technology for grid data is constructed. The public chain is a network with low access threshold. At the same time, due to the mature blockchain technology as a public chain, it has developed relatively mature. It has powerful computing barriers to protect the trustworthiness and security of blockchain data. However, the public chain has the disadvantages of a large amount of resources, consensus and low transaction speed. It avoids these shortcomings of the public chain, and integrates nonpublic chains such as private chain or alliance chain to build a reliable, efficient and low-cost hybrid for grid data. The chain protects the power data from being destroyed, reduces operating and transaction costs, and implements a multi-level data security and trusted shared network system that combines the private chain and the alliance chain with the public chain as the core.

Autonomous peer-to-peer information exchange protocol is based on blockchain architecture. The traditional power grid implements data packet interaction based on the IP routing mechanism, and exposes the location privacy of the source and destination nodes. The enterprise information exchange process carried by the blockchain is based on publish and subscribe mechanism to perform decentralized peer-to-peer information exchange of the power service system. The system Combines with the trusted identification of blockchain, the design of publish and subscribe information list and decentralized information forwarding autonomous peerto-peer exchange protocol to achieve peer-to-peer trusted exchange of power data.

\subsection{Blockchain-based electronic data depositing technology}

Electronic data deposit certificate is based on a complete set of dispute resolution mechanism. Through online one-button litigation, one-click arbitration, one-click application for notarization, traditional means of safeguarding rights are solved through Internet-based social means [4]. The system eliminates online security risks during business operations and create favourable conditions for the smooth development of the business. The unchangeable characteristics of the blockchain are in line with the requirements of electronic data storage, and the blockchain and electronic data depositing mechanism are perfectly combined.

\section{8 loT trusted access technology based on blockchain}

Trusted identification and authentication of multi-isomer Internet of Things devices in blockchain systems are constructed. In the huge IoT environment, a large number of sensing devices such as RFID, sensors, and smart tags are used in the power industry, ensuring the credibility of the data source. This is an important prerequisite for the normal operation of the system. In the case of wireless communication in the Internet of Things, an attacker can obtain the address information of the attacked by listening to the data packet of the 
attacked. The attacker launches repeated packet attacks, even pretending to be sent by the attacked, resulting in false transactions and accounting records. The diversification of equipment in the Internet of Things and the isomerization of the network have exacerbated the seriousness of the problem. Therefore, it solves the problem of easy duplication of MAC addresses, IP addresses, etc., and reliably identifies and authenticates participants (devices and people), ensures security and credibility of the data.

The system maintains consistency of transaction information and billing systems under dynamic network connections and network topologies. The connection between networks terminals (devices, users, etc.) based on the blockchain IoT system becomes highly dynamic due to the mobility of the terminal. Usually, interaction occurs only when there is a transaction such as power consumption and payment, and a short-term effective Internet of Things connection is established. Therefore, the connection of the Internet of Things system is highly dynamic [5] and uncertain.

\section{Energy blockchain technology framework and system}

In view of the challenges facing the current development of energy Internet, the key technologies of blockchain technology, key technologies such as construction implementation and application mode are proposed from the communication layer, data layer and contract layer. The energy Internet blockchain architecture is shown in Figure 1.

\subsection{Establishing a technical system for energy blockchain technology}

In view of the multi-energy coordination and multi-scale space-time coupling of the energy Internet, it is necessary to comprehensively consider the physical network constraints such as source, network, load and storage in the energy Internet. Starting from the value transmission mode and function principle of energy Internet, the technical system of energy blockchain technical specification, applicable scope, operation principle, composition structure, key technology, typical application, derivative service and organization management system coordination are constructed.

\subsection{Building a trusted and efficient hybrid chain technology for power grid}

In view of the shortcomings of the public chain, which consumes a large amount of resources and low transaction speed, the non-public chain such as private chain and alliance chain are used to balance the relationship between cost and credibility. The system constructs a hybrid chain that is reliable, efficient and low-cost for grid data, avoiding the shortage of public chain, protecting power data from being destroyed, reducing operating and transaction costs. It realizes a multi-level data security and trusted shared network system that takes the public chain as the core and combines [6] the private chain and the alliance chain.

\subsection{Implementing smart contract-based power trading technology}

For the power market, we combine with the characteristics of the blockchain and the current trading mechanism of the electricity market, design the private chain of electricity market transactions. We design the data structure and smart contracts of block for the characteristics of power trading business and transaction data, and design efficient and secure transaction data storage distribution mechanism.

\subsection{Establishing market framework system for carbon emission trading based on energy blockchain technology}

Aiming at the pain points such as quota certification and traceability of emission allowances in the carbon emission market, a market infrastructure system is built. That can meet the diversified needs of trading operations and supervision in the carbon emission market. A carbon emission market organization management system, transaction process, trading rules, transaction settlement and transaction supervision system based on energy blockchain are constructed.

\subsection{Blockchain-based electronic data depositing technology}

The system realizes real-time synchronization and preservation of electronic contract data generated by Internet marketing services, encrypted transmission, prevention of tampering, and provision of direct application for notarization after data deposit. It provides a safe and reliable evidence storage space for all parties to the transaction, and provides a quick and convenient solution once the transaction has a dispute.

\subsection{Identification and authentication technology based on biometrics and hardware features}

For the problem that the data source of the blockchain in the Internet of Things is easy to be disguised, the transaction participant identification method is constructed based on the identity of the participants, human or smart device, using biometrics or hardware features. We design multi-dimensional feature fusion algorithm to improve recognition accuracy, improve the reliability of authentication, and ensure security and credibility of the perceived data that enters the IoT environment based on blockchain.

\section{Energy blockchain application}

In this paper, we resolve the problem of energy Internet value transfer and final auditing around blockchain 


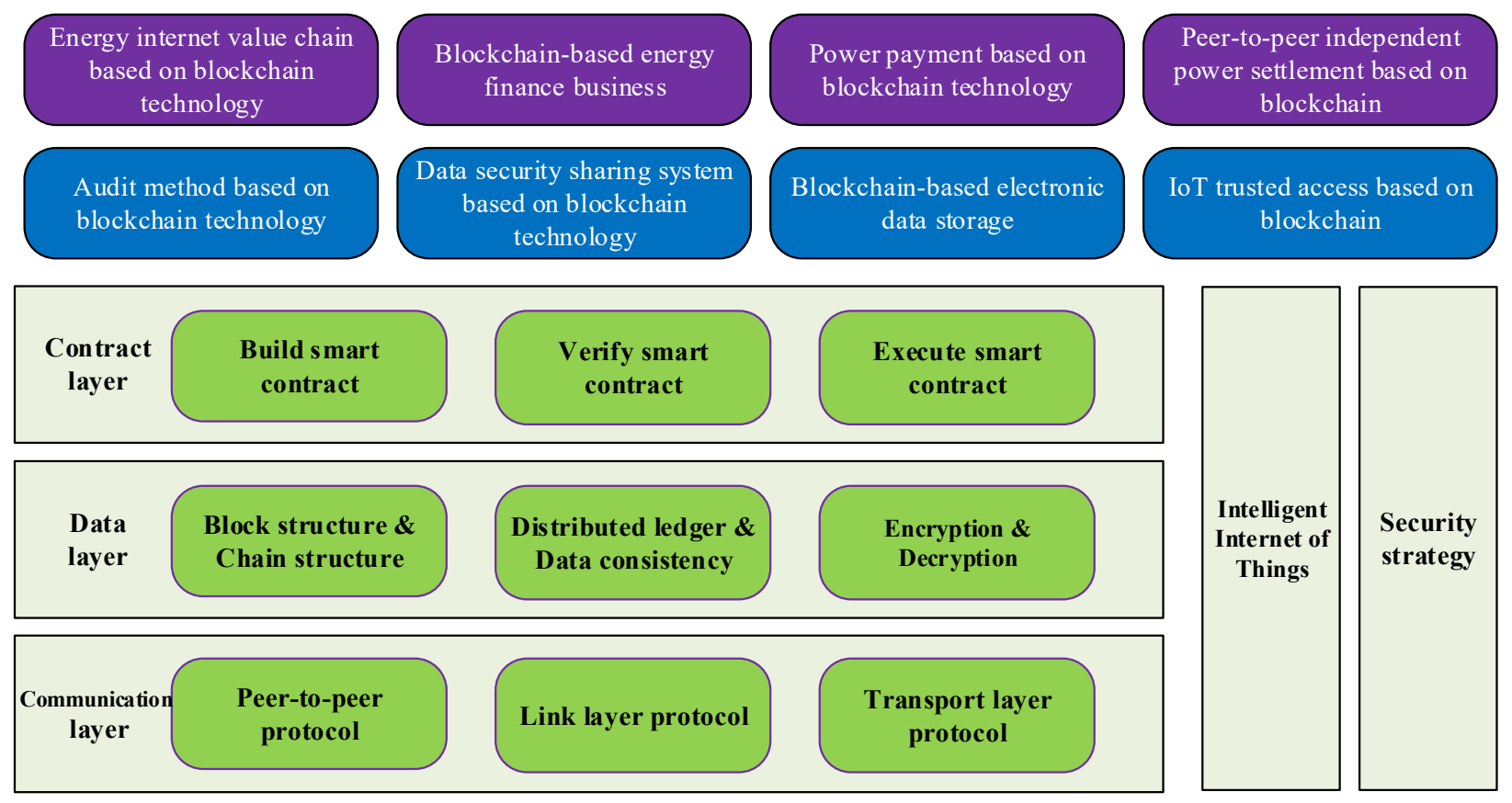

Fig. 1. Energy Internet blockchain architecture diagram.

technology, ensure information security, enhance power payment, power settlement, trust of Internet of Things, and support the development of Internet finance. The system meets the needs and provides the basis for the deep integration of the blockchain[7] and the energy Internet.

\subsection{Energy Internet value chain application based on blockchain technology}

The technical principle and value transfer of blockchain are researched, combining with the energy Internet, to build the theory and technical framework of the energy blockchain. Through the market forms such as carbon emissions trading, the circulation of energy values in the entire process of production, transmission and consumption are realized. The value circulation of multiple types of energy in the energy Internet is realized. The energy Internet value transfer model based on energy blockchain technology and its typical application are formed.

The establishment of the theory of energy blockchain involves the specification, scope, operating principle, and structural system. The key technical framework of the energy blockchain involves system architecture, core technologies, and governance systems. The core technologies involve energy data block structure, energy incentive distribution mechanism and smart contract framework. Typical application scenarios involve carbon emissions trading, photovoltaic trading and other types.

\subsection{Blockchain-based Internet financial services application}

The systems realize the core framework design of the blockchain financial system and support the upper-level Internet finance business, including guarantee services, bank lending, wealth management financing, credit reporting, and institutional supervision. Blockchain technology is used to solve the problem of data storage, verification and security assurance in traditional financial services, thus ensuring the development of the business.

Based on the development trend of internet finance in blockchain, a model combining blockchain and internet finance business is constructed [8]. It realizes the application of data storage verification and security assurance technology for internet financial services based on blockchain technology.

\subsection{Power payment enhancement application based on blockchain technology}

A zero-knowledge proof mechanism include lightning network hash key verification is used to construct a payment channel network. A peer-to-peer sharing scheme of charging piles that combines blockchain, lightning network and smart contract are constructed to realize digital asset management [9] and value security transfer.

\subsection{Peer-to-peer independent power settlement application based on blockchain}

A blockchain-based power settlement system architecture model is constructed. In this paper, we build private chain data, key technologies, blockchain data encryption technology, data security technology and smart contract technology. A smart contract model based on power transaction settlement [10] is constructed based on power currency.

From the perspective of creating power coins based on private chains, blockchain and smart contract technology are built to realize power coin creation and 
power currency-based settlement methods to improve current power transaction efficiency.

\subsection{Application of auditing method based on blockchain technology}

In this paper, we analyze the scope of application of blockchain in the internal financial settlement process of electricity, and establish a financial internal financial settlement specification based on blockchain. We establish an interface specification for existing financial systems and blockchain, improve the efficiency of final audit work, reduce the cost of final audit work, and form a power-based auditing technology based on blockchain.

Efficient methods for blockchain data retrieval based on timestamp consistency method and interface standard are established. The final audit intelligent contract model based on blockchain data and intelligent contract is constructed, and the verification method is given. From the data, storage and smart contract level, the blockchain technology supports the audit business application design.

\subsection{Data security sharing system application based on blockchain technology}

Data verification and storage technologies based on blockchain technology for power systems are established to ensure information security. The system prevents data tampering and malicious network attacks, and improve information security based on blockchain technology.

In this paper, we establish data security sharing network system based on blockchain technology, and construct key technologies for decentralized unified naming technology and services, distributed data storage and traceability methods, data encryption adaptation and efficient distribution protocols.

\subsection{Application of electronic data deposit based on blockchain}

Based on the Internet marketing service innovation, an electronic data depositing solution based on blockchain is established in the electronic data storage application scenario, suitable for the power grid. Electronic data storage application based on blockchain is formed.

Based on the blockchain-based electronic data deposit, the system is connected with the judiciary to improve efficiency and security.

\subsection{Application of loT trusted access technology based on blockchain}

In this paper, we explore the IoT application scenarios suitable for the power grid, establish IoT trusted solution based on the blockchain decentralized system, and Internet of Things interconnection and mutual trust protocol, and form the blockchain technology in the IoT application technology. We establish blockchain architecture for IoT scenarios, IoT digital intelligent terminal identification, interaction and trusted access and authentication mechanisms, and IoT transaction information distribution methods and applications based on blockchain architecture.

\section{Conclusion}

In this paper, we analyze the value distribution mode of blockchain in energy internet, integrate business form, information system, internet of things and blockchain, and build secure and autonomous peer-to-peer energy Internet environment based on blockchain.

The key technologies are constructed from the network layer, system layer and business layer. The system solves the problem of network layer equipment and data access authentication, secure and trusted sharing. It improves the system layer blockchain-based power payment, power settlement, power audit and other business support capabilities. It enhances the security and credibility of business, supports and drives the future global Energy Internet development.

\section{Acknowledgement}

This work was supported by grants from the E-commerce science and technology project of State Grid Corporation of China (No. 5100/2019-72005B), Research on Key Technology Application of Trusted Blockchain Basic Service Platform.

\section{References}

1. H. Sun, Q. Guo, Z. Pan. Energy internet: concept architecture and frontier outlook. Automation of Electric Power Systems 39.19 (2015): 1-8.

2. J. Dai, and A. V. Miklos. Toward blockchain-based accounting and assurance. Journal of Information Systems 31.3 (2017): 5-21..

3. A. Bahga, V. K. Madisetti. Blockchain platform for industrial internet of things. Journal of Software Engineering and Applications 9.10 (2016): 533.

4. S. N. Shah. Device-driven non-intermediated blockchain system over a social integrity network. U.S. Patent Application No. 15/372,699 (2017).

5. S. Feng, P. Setoodeh, S. Haykin. Smart home: Cognitive interactive people-centric Internet of Things. IEEE Communications Magazine $\mathbf{5 5 . 2}$ (2017): 34-39.

6. L. Wu, K. Meng, S. Xu, et al. Democratic centralism: A hybrid blockchain architecture and its applications in energy internet. IEEE International Conference on Energy Internet (ICEI). IEEE, (2017): 176-181.

7. X. Tai, H. Sun, Q. Guo. Electricity transactions and congestion management based on blockchain in energy internet. Power Syst. Technol 40 (2016): 3630-3638.

8. H. Wang, K. Chen, D. Xu. A maturity model for blockchain adoption. Financial Innovation $\mathbf{2 . 1}$ (2016): 12. 
9. J. Mendling, I. Weber, W. V. D. Aalst, et al. Blockchains for business process managementchallenges and opportunities. ACM Transactions on Management Information Systems (TMIS) $\mathbf{9 . 1}$ (2018): 4.

10. S. Zhao, B. Wang, Y. Li, et al. Integrated Energy Transaction Mechanisms Based on Blockchain Technology. Energies 11.9 (2018): 2412. 